\title{
Exact Green's function for rectangular potentials and its application to quasi-bound states
}

\author{
Fabiano M. Andrade ${ }^{\mathrm{a}}$ \\ ${ }^{a}$ Departamento de Matemática e Estatística, Universidade Estadual de Ponta Grossa, 84030-900 Ponta Grossa-PR, Brazil
}

\begin{abstract}
In this work we calculate the exact Green's function for arbitrary rectangular potentials. Specifically we focus on Green's function for rectangular quantum wells enlarging the knowledge of exact solutions for Green's functions and also generalizing and resuming 'results in the literature. The exact formula has the form of a sum over paths and always can be cast into a closed analytic expression. From the poles and residues of the Green's function the bound states eigenenergies and eigenfunctions with the correct normaliza'tion constant are obtained. In order to show the versatility of the method, an application of the Green's function approach to extract information of quasi-bound states in rectangular barriers, where the standard analysis of quantum amplitudes fail, is presented.
\end{abstract}

Keywords: rectangular potential, Green's function, bound state, resonant scattering

\section{Introduction}

One-dimensional systems are used frequently in quantum mechanics to approximate real situations because they are rel'atively easy to treat mathematically, allowing to get a deeper insight on the physics involved. They are useful in a number of applications in contemporary physics and their simplicity has made them valuable as academic and research tools. For instance, it turns up in the description of resonant tunnel'ing diode devices, disordered one-dimensional lattices, realistic one-dimensional solid-state system such as quantum wells, 'junctions and superlattices $[1,2]$, and time analysis of onedimensional tunneling processes [3]. In particular, one-dimen'sional rectangular potentials can be used to model isolated tran'sitions, observed in semiconductors, from a bound state within 'a quantum well to a bound state at an energy greater than the barrier height [4]. Limiting cases of rectangular potentials can 'be used to describe point interactions [5], as well as regularized 'singular interactions [6]. Also, we should point out that the use 'of square-barrier and square-well potentials as simple models for more realistic physical problems has a long history in the 'theory of heterostructures in solid-state physics [7].

However, the propagator or its Fourier transform, the Green's function, for rectangular potentials, although these are very simple systems, are difficult to obtain and involve lengthy and tedious calculations. Indeed, the exact Green's function and propagators for step potential and square barrier was obtained only in the late 1980s until the early 1990s [8-12]. Moreover, it is well known that semiclassical approach, i.e., the Van VleckGutzwiller formula [13], or the WKB approach [14], give poor results when applied to the class of rectangular potentials due to their discontinuities.

In the present work, we show how to obtain the exact Green's function [15-17], given as a general sum over paths, in a very simple way avoiding complicated calculations, for arbitrary rectangular potentials enlarging the knowledge of exact solutions for Greens functions and also generalizing and resuming results in the literature. The procedure outlined here can be thought of as exact version of the approximation in [18] and provides simple and direct way to construct recurrence relations for quantum amplitudes for one-dimensional scattering discussed in [19]. Specifically, we focus on Green's function for single quantum wells to avoid extra and unnecessary mathematical complications, but the method is general and could be applied to multiple quantum wells as well [17]. From the Green's functions obtained, we thus describe how to extract the bound states eigenenergies and eigenfunctions. Also, we discuss an application of the Green's function approach to extract information of quasibound states in rectangular potentials. We should observe that the Green's function approach used in the present work, has been successfully used in the calculation of exact Green's functions for segmented potentials [15], in calculation of asymptotic Green's functions [16], in the determination and discussion of bound states in multiple quantum wells [17]. Also, in the calculation of exact Green's function for quantum graphs [20], general point interactions [21], and scattering quantum walks [22, 23].

This paper is organized as follows. In Sec. 2, we give a briefly review on the definition and properties of the Green's functions. In Sec. 3, the Green's functions approach for the rectangular potential is presented. In Sec. 4, the construction of the exact Green's functions for a general rectangular asymmetric well potential is presented and how the sought eigenenergies and eigenfunctions are extracted. In Sec. 5, the case of the square well potential is discussed and the definite parity eigenfunctions are determined. In Sec. 6 the case of the infinite well potential is discussed. In Sec. 7 a Green's function approach to extract information from system with quasi-bound states is presented. Finally, Sec. 8 contains our conclusions.

Email address: fmandrade@uepg. br (Fabiano M. Andrade) 


\section{Green's function: Definition and properties}

The Green's functions are an important tool in quantum mechanics for calculating eigenenergies, eigenfunctions and density of states [24]. It can be defined as solutions of the inhomogeneous differential equation

$$
\left[E-\hat{H}\left(x_{f}\right)\right] G\left(x_{f}, x_{i} ; E\right)=\delta\left(x_{f}-x_{i}\right),
$$

subject to certain boundary conditions. $\hat{H}(x)$ is the Hamiltonian operator

$$
\hat{H}(x)=-\frac{\hbar^{2}}{2 m} \frac{d^{2}}{d x^{2}}+V(x) .
$$

The Green's function $G\left(x_{f}, x_{i} ; E\right)$ for a quantum system can be interpreted as a probability amplitude for a particle that initially at the point $x_{i}$ moves to the point $x_{f}$ for a fixed energy [25]

$$
G\left(x_{f}, x_{i} ; E\right)=\left\langle x_{f}|\hat{G}(E)| x_{i}\right\rangle,
$$

where $\hat{G}(E) \equiv \lim _{\eta \rightarrow 0^{+}}[E-\hat{H}(x)+i \eta]^{-1}$ is the resolvent operator [26].

Suppose we have a complete set of normalized energy eigenstates $\psi_{n}(x), n=0,1, \ldots$ with

$$
\hat{H}(x) \psi_{n}=E_{n} \psi_{n},
$$

so that the solution of Eq. (1) can be written as

$$
G\left(x_{f}, x_{i} ; E\right)=\sum_{n} \frac{\psi_{n}\left(x_{f}\right) \psi_{n}^{*}\left(x_{i}\right)}{E-E_{n}} .
$$

For a discrete spectrum, we thus identify the poles of Green's function with bound states eigenenergies and the residues at these points give a tensorial product of the bound states eigenfunctions. The continuous part of the spectrum corresponds to a branch cut of $G\left(x_{f}, x_{i} ; E\right)[26,27]$. The following limit

$$
\lim _{E \rightarrow E_{n}}\left(E-E_{n}\right) G\left(x_{f}, x_{i} ; E\right)=\psi_{n}\left(x_{f}\right) \psi_{n}^{*}\left(x_{i}\right)
$$

is used to extract the discrete bound states from the Green's function.

There are basically three methods for calculating the Green's functions [24]: solving the differential equation in (1); summing up the spectral representation in (5); or performing the Feynman path integral expansion for the Green's function [28]. In this paper, we present a Feynman-like procedure. As we are going to see, the approach requires one to determine the quantum amplitudes of each individual potential, but in general, this is a much easier task than to calculate numerically propagation along the whole $V(x)$.

\section{The Green's function approach for rectangular poten- tials}

According to Refs. [15-17] the Green's function $G\left(x_{f}, x_{i} ; E\right)$ (in what follow, we will denote it by $G_{f, i}$ for short) for a general $1 \mathrm{D}$ potential is obtained by writing the potential $V(x)$ as a sum of $n$ individual potentials $u_{j}$, where each $u_{j}$ vanish asymptotically. In the present work, we focus our attention on rectangular

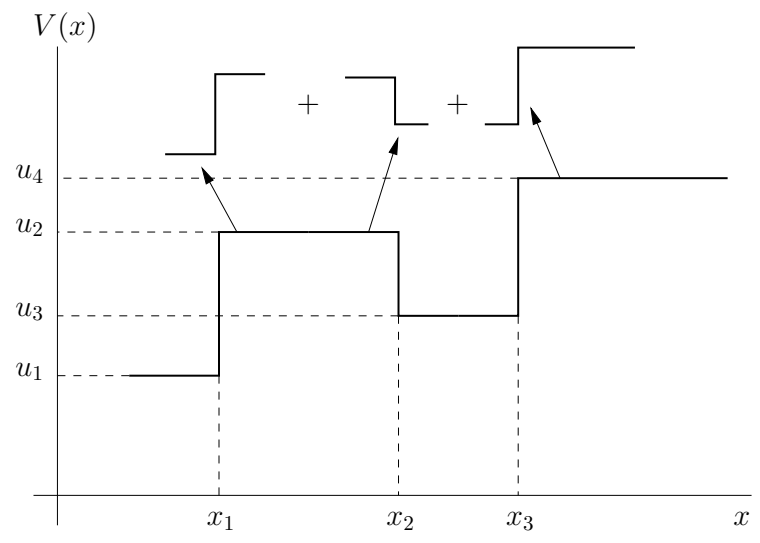

Figure 1: A rectangular potential with three points of discontinuities. The potential can be considered as a sum of the three potential steps.

potentials. As depicted in Fig. 1, a rectangular potential can be treated as the sum of $n$ step potentials. The potential function for a rectangular potential can be written as

$$
V(x)=\left\{\begin{array}{lll}
u_{1} \text { for } x<x_{1}, & (\text { Region } 1), \\
u_{j} \text { for } x_{j} \leq x<x_{j+1}=x_{j}+\ell_{j}, & (\text { Region } j), \\
u_{n} \text { for } x \geq x_{n}, & (\text { Region } n),
\end{array}\right.
$$

where $u_{j}, \ell_{j}$ and $x_{j}$ are the height of the potential, the length of the region $j$ and the localization of the $j$ th discontinuous point, respectively. The wave number in each different region is $k_{j}=$ $\sqrt{2 m\left(E-u_{j}\right) / \hbar}=i \kappa_{j}$. In what follow, we do not distinguish the cases whether $k_{j}$ is a real or a purely imaginary number $\left(i \kappa_{j}\right)$, except otherwise mentioned.

The Green's function for this system, for a fixed energy $E$ and the end points $x_{i}$ and $x_{f}$, is given by

$$
G_{f, i}=\frac{m}{i \hbar^{2} \sqrt{k_{f} k_{i}}} \sum_{\mathrm{sp}} W_{\mathrm{sp}} \exp \left[\frac{i}{\hbar} S_{\mathrm{sp}}\left(x_{f}, x_{i} ; E\right)\right],
$$

where $W_{\mathrm{sp}}$ is the amplitude (or weight) and $S_{\mathrm{sp}}$ is the classical action. The above sum is performed over all scattering paths (sp) starting in $x_{i}$ and ending in $x_{f}$. For each sp, the classical action is obtained from the propagation over action of potential $u_{j}, S_{\mathrm{sp}}\left(x_{b}, x_{a} ; E\right)=\hbar \int_{x_{a}}^{x_{b}} k_{j} d x$. A few comments concerning the Eq. (8) are necessary. The expression in Eq. (8) is obtained from a recursive procedure, i.e., $G_{f, i}$ for $n$ potentials are derived from $G_{f, i}$ for $n-1$ potentials. The $W_{\mathrm{sp}}$ are related to local quantum effects, so they depend on quantum amplitudes $\left(R_{j}^{( \pm)}\right.$and $T_{j}^{( \pm)}$) of individual $u_{j}$ (cf. Appendix A). In fact, for the present case of rectangular potentials, $R_{j}^{( \pm)}$and $T_{j}^{( \pm)}$are the usual reflection and transmission amplitudes of individual $u_{j}$ [19] (up to a phase [15]). (The superscript $(+)((-))$ and subscript $j$ denote the physical quantities of a quantum particle incident from left (right) at the point $x_{j}$, respectively.) In the general case of smooth potentials, they are related, but we need to calculate classical actions for each potential. In this later case, the interested reader can consult the Refs. [16, 17] for derivations. 


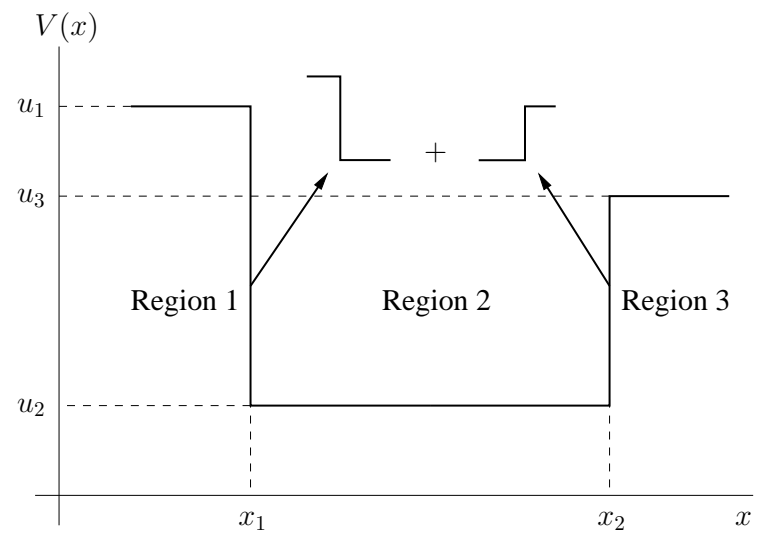

Figure 2: An asymmetric well potential written as a sum of a left and right potential steps.

\section{Asymmetric well potential}

In this section we will consider the case of a quantum particle confined in the asymmetric well potential defined by

$$
V(x)=\left\{\begin{array}{lll}
u_{1} \text { for } x<x_{1}, & (\text { Region } 1), \\
u_{2} \text { for } x_{1} \leq x<x_{2}=x_{1}+\ell_{2}, & (\text { Region 2) } \\
u_{3} \text { for } x \geq x_{2}, & (\text { Region 3) }
\end{array}\right.
$$

and depicted in Fig. 2. We seek the Green's function for the situation where $E<u_{3}$. The eigenvalues will be obtained from the poles of the Green's function and the eigenfunctions from their respective residues (cf. Sec. 2). We can write nine different Green's functions for the problem, but for our purposes, we analyze only three relevant possibilities, namely: (1) $x_{1}<x_{i}<x_{2}$ and $x_{f}<x_{1}$; (2) $x_{1}<x_{i}, x_{f}<x_{2}$; and (3) $x_{1}<x_{i}<x_{2}$ and $x_{f}>x_{2}$. The others six Green's functions can be obtained from symmetry considerations of the three above cases.

First, we want to exemplify how to use the Eq. (8) and how the geometric series appear. So, let us consider the possibility (2). To obtain $G_{f, i}^{(2)}$ in a closed form, we need to sum up all the possibles scattering paths for a quantum particle starting from $x_{i}$ and arriving at $x_{f}$, but it can always be done because the sum in (8) forms a geometric series. Without loss of generality, let us set $x_{f}>x_{i}$.

Thus, in Fig. 3 is depicted five examples of scattering paths. The first path in Fig. 3(a) is the direct propagation from $x_{i}$ to $x_{f}$, which contributes with $e^{i k_{2}\left(x_{f}-x_{i}\right)}$ for $G_{f, i}^{(2)}$. In Fig. 3(b), a quantum particle leaves the point $x_{i}$, goes to the point $x_{2}$, where it hit the potential and is reflected, and so, goes to left to arrives at the point $x_{f}$. This path contributes with $e^{i k_{2}\left(x_{2}-x_{i}\right)} R_{2}^{(+)} e^{-i k_{2}\left(x_{f}-x_{2}\right)}$, where $R_{2}^{(+)}$is the reflection amplitude for a quantum particle incident from the left of the potential $u_{3}$ at the point $x_{2}$. Following the same reasoning, the contributions for the other examples in Fig. 3 are:

$$
\begin{aligned}
& \text { (c) } e^{-i k_{2}\left(x_{1}-x_{i}\right)} R_{1}^{(-)} e^{i k_{2}\left(x_{f}-x_{1}\right)} \\
& \text { (d) } e^{i k_{2}\left(x_{2}-x_{i}\right)} R_{2}^{(+)} e^{i k_{2} \ell_{2}} R_{1}^{(-)} e^{i k_{2}\left(x_{f}-x_{1}\right)} \\
& \text { (e) } e^{-i k_{2}\left(x_{1}-x_{i}\right)} R_{1}^{(-)} e^{i k_{2} \ell_{2}} R_{2}^{(+)} e^{-i k_{2}\left(x_{f}-x_{2}\right)}
\end{aligned}
$$

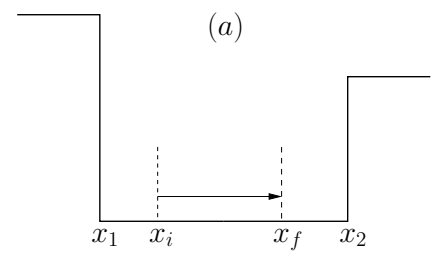

(c)

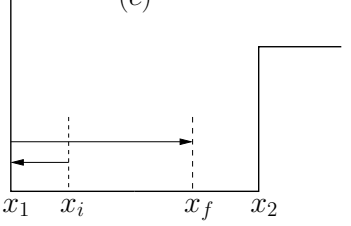

(e)
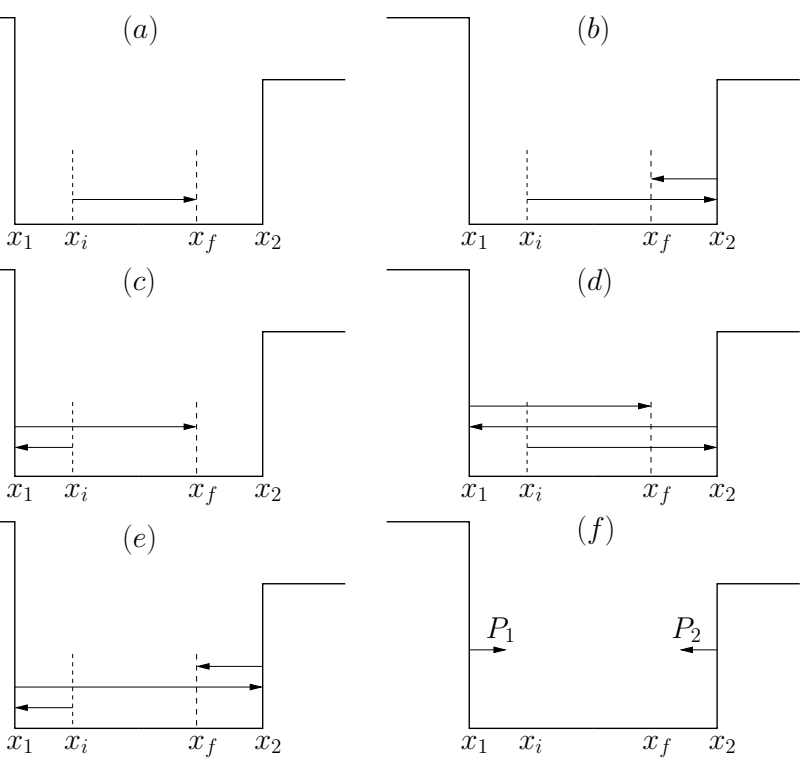

(d)

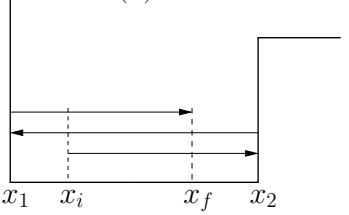

$(f)$

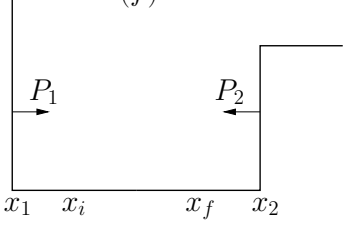

Figure 3: In (a)-(e) schematic examples of scattering paths and in (f) the families of paths $P_{1}$ and $P_{2}$.

The Green's function is then given by the sum of all contributions of $n$ multiple reflections of all possibles scattering paths and can be written as $\left(\ell_{2}=x_{2}-x_{1}\right)$

$$
\begin{aligned}
G_{f, i}^{(2)}= & \frac{m}{i k_{2} \hbar^{2}}\left\{e^{i k_{2}\left(x_{f}-x_{i}\right)}\right. \\
& +\sum_{n=0}^{\infty}\left(R_{1}^{(-)} R_{2}^{(+)} e^{2 i k_{2} \ell_{2}}\right)^{n}\left[e^{-i k_{2}\left(x_{1}-x_{i}\right)} R_{1}^{(-)}\right. \\
& \times\left(e^{i k_{2}\left(x_{f}-x_{1}\right)}+R_{2}^{(+)} e^{i k_{2} \ell_{2}} e^{-i k_{2}\left(x_{f}-x_{2}\right)}\right) \\
& +e^{i k_{2}\left(x_{2}-x_{i}\right)} R_{2}^{(+)} \\
& \left.\left.\times\left(e^{-i k_{2}\left(x_{f}-x_{2}\right)}+R_{1}^{(-)} e^{i k_{2} \ell_{2}} e^{i k_{2}\left(x_{f}-x_{1}\right)}\right)\right]\right\} .
\end{aligned}
$$

In general, the reflections (transmissions) amplitudes have the property $\left|R_{j}^{( \pm)}\right|^{2} \leq 1\left(\left|T_{j}^{( \pm)}\right|^{2} \leq 1\right)$, in such way as the above sum always converge. In fact, it is a geometric series. So, after a straightforward algebra the final form for the Green's function is

$$
\begin{aligned}
G_{f, i}^{(2)}= & \frac{m}{i \hbar^{2} k_{2} f_{\mathrm{aw}}}\left(e^{i k_{2}\left(x_{f}-x_{1}\right)}+R_{2}^{(+)} e^{i k_{2} \ell_{2}} e^{i k_{2}\left(x_{2}-x_{f}\right)}\right) \\
& \times\left(e^{-i k_{2}\left(x_{i}-x_{1}\right)}+R_{1}^{(-)} e^{i k_{2} \ell_{2}} e^{-i k_{2}\left(x_{2}-x_{i}\right)}\right),
\end{aligned}
$$

where $f_{\text {aw }}=1-R_{1}^{(-)} R_{2}^{(+)} e^{2 i k_{2} \ell_{2}}$.

The method utilized above consists in sum up all scattering paths, but this could be very tedious and cumbersome. However, this can be done by a simple diagrammatic classification in families of paths, which is a practical way to identify and perform the geometric series mentioned above. Consider again the confined particle in the asymmetric well potential. The particle starting from $x_{i}$ may (a) go to the right arriving $x_{f}$; or (b) go to the left, hit the potential $u_{1}$ at $x_{1}$ being reflected. There is an infinite family of paths for the particle at $x_{1}$, we call this family $P_{1}$; and (c) the particle go to the right, hit the potential $u_{3}$ at $x_{2}$ being reflected. Like before, there is infinite family of 
paths for the particle at $x_{2}$, we call this family $P_{2}$. These two infinite family of paths are schematically depicted in Fig. 3(f). Thus, by using the above prescription, the Green's function can be written as

$$
\begin{aligned}
G_{f, i}^{(2)}= & \frac{m}{i \hbar^{2} k_{2}}\left(e^{i k_{2}\left(x_{f}-x_{i}\right)}\right. \\
& \left.+e^{i k_{2}\left(x_{2}-x_{i}\right)} R_{2}^{(+)} P_{2}+e^{-i k_{2}\left(x_{1}-x_{i}\right)} R_{1}^{(-)} P_{1}\right) .
\end{aligned}
$$

The family $P_{1}\left(P_{2}\right)$ posses two contributions: (a) go to the right (left), arriving at $x_{f}$; and (b) go to the right (left), hit the potential $u_{3}\left(u_{1}\right)$ at $x_{2}\left(x_{1}\right)$ being reflected followed by the family $P_{2}$ $\left(P_{1}\right)$. Thus,

$$
\begin{aligned}
& P_{1}=e^{i k_{2}\left(x_{f}-x_{1}\right)}+e^{i k_{2} \ell_{2}} R_{2}^{(+)} P_{2}, \\
& P_{2}=e^{i k_{2}\left(x_{f}-x_{2}\right)}+e^{i k_{2} \ell_{2}} R_{1}^{(-)} P_{1} .
\end{aligned}
$$

Solving for $P_{1}$ and $P_{2}$ one obtains

$$
\begin{aligned}
& P_{1}=\frac{1}{f_{\mathrm{aw}}}\left(e^{i k_{2}\left(x_{f}-x_{1}\right)}+R_{2}^{(+)} e^{i k_{2} \ell_{2}} e^{i k_{2}\left(x_{2}-x_{f}\right)}\right), \\
& P_{2}=\frac{1}{f_{\mathrm{aw}}}\left(e^{i k_{2}\left(x_{2}-x_{f}\right)}+R_{1}^{(-)} e^{i k_{2} \ell_{2}} e^{i k_{2}\left(x_{f}-x_{1}\right)}\right) .
\end{aligned}
$$

By substitution of Eq. (14) into Eq. (12), one obtains the Green's function in Eq. (11). The other two cases can be obtained in a similar way. For instance, for case (1) we have

$$
\begin{aligned}
G_{f, i}^{(1)}= & \frac{m}{i \hbar^{2} \sqrt{k_{1} k_{2}} f_{\mathrm{aw}}}\left(T_{1}^{(-)} e^{-i k_{1}\left(x_{f}-x_{1}\right)}\right) \\
& \times\left(e^{-i k_{2}\left(x_{i}-x_{1}\right)}+R_{1}^{(-)} e^{i k_{2} \ell_{2}} e^{-i k_{2}\left(x_{2}-x_{i}\right)}\right),
\end{aligned}
$$

and for case (3), we obtain

$$
\begin{aligned}
G_{f, i}^{(3)}= & \frac{m}{i \hbar^{2} \sqrt{k_{3} k_{2}} f_{\mathrm{aw}}}\left(T_{2}^{(+)} e^{i k_{3}\left(x_{f}-x_{2}\right)}\right) \\
& \times\left(e^{-i k_{2}\left(x_{i}-x_{1}\right)}+R_{1}^{(-)} e^{i k_{2} \ell_{2}} e^{-i k_{2}\left(x_{2}-x_{i}\right)}\right) .
\end{aligned}
$$

\subsection{Calculation of the bound states}

The bound states are calculated from the residues of the Green's functions in Eqs. (11), (15) and (16). Its poles $E_{n}=$ $\hbar^{2} k_{n}^{2} / 2 m$ are all contained in the term $1 / f_{\text {aw }}$. They are calculated from $f_{\text {aw }}=0$, which leads to the following transcendental equation

$$
\left(\frac{k_{2}^{(n)}-k_{1}^{(n)}}{k_{1}^{(n)}+k_{2}^{(n)}}\right)\left(\frac{k_{2}^{(n)}-k_{3}^{(n)}}{k_{2}^{(n)}+k_{3}^{(n)}}\right) e^{2 i k_{2}^{(n)} \ell_{2}}=1,
$$

where $k_{j}^{(n)}=\sqrt{2 m\left(E_{n}-u_{j}\right)} / \hbar$. This result agree with the one found by the solution of the Schrödinger equation [29]. Using the formula

$$
\lim _{E \rightarrow E_{n}} \frac{E-E_{n}}{f_{\text {aw }}}=\frac{\hbar^{2}}{2 m} \lim _{k \rightarrow k_{n}} \frac{k^{2}-k_{n}^{2}}{f_{\text {aw }}}=\frac{\hbar^{2}}{m} \frac{k_{n}}{f_{\text {aw }}^{\prime(n)}},
$$

where $f_{\text {aw }}^{\prime(n)}=\left.\left(d f_{\text {aw }} / d k\right)\right|_{k=k_{n}}$, we obtain for the residues of the Green's function in Eqs. (11), (15) and Eq. (16)

$$
\begin{aligned}
& \psi_{n}^{(2)}\left(x_{f}\right)\left[\psi_{n}^{(2)}\left(x_{i}\right)\right]^{*}=\frac{\hbar^{2}}{2 m} \lim _{k \rightarrow k_{n}}\left(k^{2}-k_{n}^{2}\right) G_{f, i}^{(2)} \\
& =\frac{1}{i f_{\mathrm{aw}}^{\prime(n)}}\left[e^{i k_{2}^{(n)}\left(x_{f}-x_{1}\right)}+\frac{k_{2}^{(n)}-k_{3}^{(n)}}{k_{2}^{(n)}+k_{3}^{(n)}} e^{i k_{2}^{(n)}\left(\ell_{2}+x_{2}-x_{f}\right)}\right] \\
& \times\left[e^{-i k_{2}^{(n)}\left(x_{i}-x_{1}\right)}+\frac{k_{2}^{(n)}-k_{1}^{(n)}}{k_{1}^{(n)}+k_{2}^{(n)}} e^{i k_{2}^{(n)}\left(\ell_{2}-x_{2}+x_{i}\right)}\right], \\
& \psi_{n}^{(1)}\left(x_{f}\right)\left[\psi_{n}^{(2)}\left(x_{i}\right)\right]^{*}=\frac{\hbar^{2}}{2 m} \lim _{k \rightarrow k_{n}}\left(k^{2}-k_{n}^{2}\right) G_{f, i}^{(2)} \\
& =\frac{1}{i f_{\mathrm{aw}}^{\prime(n)}}\left[\sqrt{\frac{k_{1}^{(n)}}{k_{2}^{(n)}}} \frac{2 k_{2}^{(n)}}{k_{1}^{(n)}+k_{2}^{(n)}} e^{-i k_{1}^{(n)}\left(x_{f}-x_{1}\right)}\right] \\
& \times\left[e^{-i k_{2}^{(n)}\left(x_{i}-x_{1}\right)}+\frac{k_{2}^{(n)}-k_{1}^{(n)}}{k_{1}^{(n)}+k_{2}^{(n)}} e^{i k_{2}^{(n)}\left(\ell_{2}-x_{2}+x_{i}\right)}\right], \\
& \psi_{n}^{(3)}\left(x_{f}\right)\left[\psi_{n}^{(2)}\left(x_{i}\right)\right]^{*}=\frac{\hbar^{2}}{2 m} \lim _{k \rightarrow k_{n}}\left(k^{2}-k_{n}^{2}\right) G_{f, i}^{(3)} \\
& =\frac{1}{i f_{\mathrm{aw}}^{\prime(n)}}\left[\sqrt{\frac{k_{3}^{(n)}}{k_{2}^{(n)}}} \frac{2 k_{2}^{(n)}}{k_{2}^{(n)}+k_{3}^{(n)}} e^{i k_{3}^{(n)}\left(x_{f}-x_{2}\right)}\right] \\
& \times\left[e^{-i k_{2}^{(n)}\left(x_{i}-x_{1}\right)}+\frac{k_{2}^{(n)}-k_{1}^{(n)}}{k_{1}^{(n)}+k_{2}^{(n)}} e^{i k_{2}^{(n)}\left(\ell_{2}-x_{2}+x_{i}\right)}\right] .
\end{aligned}
$$

The correct normalized eigenfunctions corresponding to the bound states of the system are thus given by:

Region 1: $x<x_{1}$

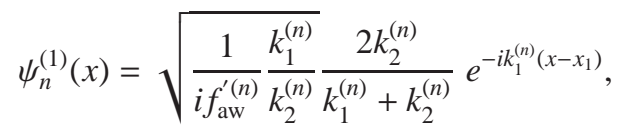

Region 2: $x_{1}<x<x_{2}$

$$
\begin{aligned}
\psi_{n}^{(2)}(x)= & \sqrt{\frac{1}{i f_{\mathrm{aw}}^{\prime(n)}}\left(e^{i k_{2}^{(n)}\left(x-x_{1}\right)}\right.} \\
& \left.++\frac{k_{2}^{(n)}-k_{3}^{(n)}}{k_{2}^{(n)}+k_{3}^{(n)}} e^{i k_{2}^{(n)}\left(\ell_{2}+x_{2}-x\right)}\right),
\end{aligned}
$$

Region 3: $x>x_{2}$

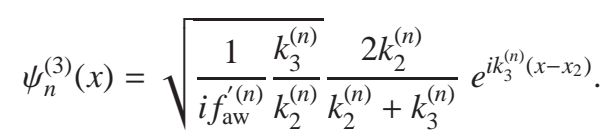

It should be observed that $k_{2}^{(n)}\left(k_{1}^{(n)}\right.$ and $\left.k_{3}^{(n)}\right)$ is (are) real (imaginary) number(s), and consequently $\psi_{n}^{(2)}(x)\left(\psi_{n}^{(1)}(x)\right.$ and $\left.\psi_{n}^{(3)}(x)\right)$ is (are) an oscillatory complex (decreasing real) exponential function(s) of $x$. Also, the Green's function used here yields the correct normalization constant which often involves a difficult integral in the other methods. In what follow, we apply the results obtained in this section in other well-known rectangular potentials. 


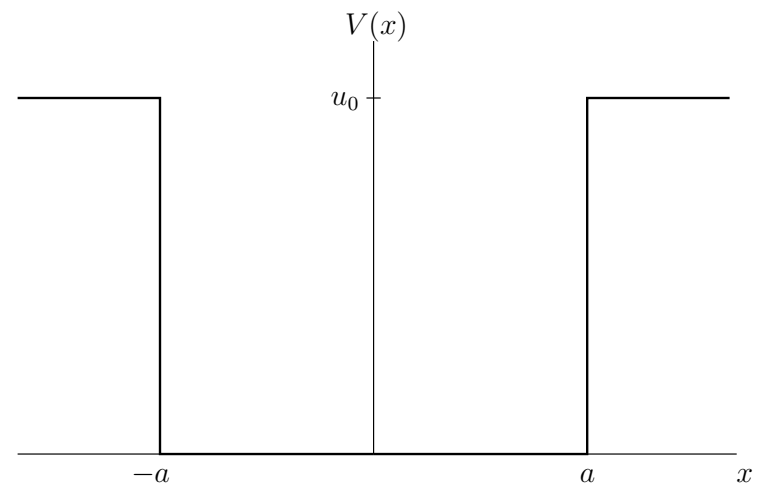

Figure 4: A square well potential.

\section{Square well potential}

The Green's function for a square well potential (depicted in Fig. 4) can be obtained from the results of the previous section by setting $u_{1}=u_{3}=u_{0}$ and $u_{2}=0$, thus $k_{1}=k_{3}=k_{0}$ and $k_{2}=k=\sqrt{2 m E} / \hbar$, also $x_{1}=-a$ and $x_{2}=a$. From Eqs. (11), (15) and (16) we thus find

$$
\begin{aligned}
G_{f, i}^{(1)}= & \frac{m}{i \hbar^{2} \sqrt{k_{0} k} f_{\mathrm{sw}}}\left(T_{(-a)}^{(-)} e^{-i k_{0}\left(x_{f}+a\right)}\right) \\
& \times\left(e^{-i k\left(x_{i}+a\right)}+R_{(-a)}^{(-)} e^{2 i k a} e^{-i k\left(a-x_{i}\right)}\right), \\
G_{f, i}^{(2)}= & \frac{m}{i \hbar^{2} k f_{\mathrm{sw}}}\left(e^{i k\left(x_{f}+a\right)}+R_{(a)}^{(+)} e^{2 i k a} e^{i k\left(a-x_{f}\right)}\right) \\
& \times\left(e^{-i k\left(x_{i}+a\right)}+R_{(-a)}^{(-)} e^{2 i k a} e^{-i k\left(a-x_{i}\right)}\right), \\
G_{f, i}^{(3)}= & \frac{m}{i \hbar^{2} \sqrt{k_{0} k} f_{\mathrm{sw}}}\left(T_{(a)}^{(+)} e^{i k_{0}\left(x_{f}-a\right)}\right) \\
& \times\left(e^{-i k\left(x_{i}+a\right)}+R_{(-a)}^{(-)} e^{2 i k a} e^{-i k\left(a-x_{i}\right)}\right),
\end{aligned}
$$

where $f_{\mathrm{sw}}=1-R_{(-a)}^{(-)} R_{(a)}^{(+)} e^{4 i k a}$.

\subsection{Calculation of the bound states}

The eigenenergies are calculated from the condition $f_{\mathrm{sw}}=$ 0 . By considering the symmetry of the potential, we can infer that the reflections amplitudes are equal (up to a phase), i.e., $R_{(-a)}^{(-)}=R_{(a)}^{(+)}=\left(k-k_{0}\right) /\left(k+k_{0}\right)$, leading to the following transcendental equation

$$
\left(\frac{k_{n}-k_{0}^{(n)}}{k_{n}+k_{0}^{(n)}}\right)^{2} e^{4 i k_{n} a}=1
$$

Using Eq. (18) the residues are determined and leads to the following bound state eigenfunctions:

Region 1: $x<-a$

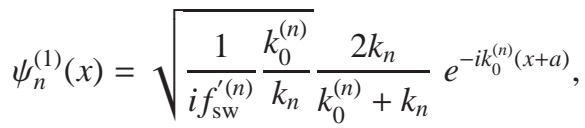

Region 2: $-a<x<a$

$$
\begin{aligned}
\psi_{n}^{(2)}(x)= & \sqrt{\frac{1}{i f_{\mathrm{sw}}^{\prime(n)}}}\left(e^{i k_{n}(x+a)}\right. \\
& \left.+\frac{k_{n}-k_{0}^{(n)}}{k_{n}+k_{0}^{(n)}} e^{2 i k_{n} a} e^{i k_{n}(a-x)}\right),
\end{aligned}
$$

Region 3: $x>a$

$$
\psi_{n}^{(3)}(x)=\sqrt{\frac{1}{i f_{\mathrm{sw}}^{\prime(n)}} \frac{k_{0}^{(n)}}{k_{n}}} \frac{2 k_{n}}{k_{n}+k_{0}^{(n)}} e^{i k_{0}^{(n)}(x-a)} .
$$

From Eq. (25), we can see that there are two equations describing the eigenenergies. These eigenenergies are determined by solving

$$
\begin{array}{lc}
\left(k_{n}-k_{0}^{(n)}\right) e^{2 i k_{n} a}=+\left(k_{n}+k_{0}^{(n)}\right), & (\text { even parity) } \\
\left(k_{n}-k_{0}^{(n)}\right) e^{2 i k_{n} a}=-\left(k_{n}+k_{0}^{(n)}\right), & \text { (odd parity) }
\end{array}
$$

and the respective eigenfunctions are

$$
\psi_{n}^{(2)}(x)=2 \sqrt{\frac{1}{i f_{\mathrm{sw}}^{\prime(n)}}} \begin{cases}e^{i k_{n} a} \cos \left[k_{n} x\right], & \text { (even parity), } \\ i e^{i k_{n} a} \sin \left[k_{n} x\right], & \text { (odd parity), }\end{cases}
$$

which are the well-known even and odd solutions for the square well potential [30].

\section{Infinite well potential}

In this section, we consider the infinite well potential. Similarly as in previous sections, the Green's function for the infinite well potential can be obtained by letting $u_{1}=u_{3}=\infty$ and $x_{1}=0$ and $x_{2}=L$, so that the potential function is given by

$$
V(x)= \begin{cases}0 & \text { for } 0<x<L, \\ \infty & \text { otherwise }\end{cases}
$$

As the potentials are infinite in $x_{1}$ and $x_{2}$, the reflection amplitude assume the value $R_{(0)}^{(-)}=R_{(L)}^{(+)}=-1$ and a vanishing transmission amplitude. Thus, the Green's function in Regions 1 and 3 vanishes, so that $\psi_{n}^{(1)}(x)=\psi_{n}^{(3)}(x)=0$. The Green's function in Region 2 take the form

$$
\begin{aligned}
G_{f, i}^{(2)}= & \frac{m}{i \hbar^{2} k f_{\mathrm{iw}}}\left(e^{i k x_{f}}-e^{i k L} e^{i k\left(L-x_{f}\right)}\right) \\
& \times\left(e^{-i k x_{i}}-e^{i k L} e^{-i k\left(L-x_{i}\right)}\right)
\end{aligned}
$$

with $f_{\text {iw }}=1-e^{2 i k L}$. After a straightforward algebra, the Eq. (31) can be written as

$$
G_{f, i}^{(2)}=\frac{2 m}{\hbar^{2} k \sin [k L]} \sin \left[k\left(x_{f}-L\right)\right] \sin \left[k x_{i}\right] .
$$

This result is indeed the exact one obtained through the spectral expansion of the Green's function in Eq. (5) (cf. Appendix B). 


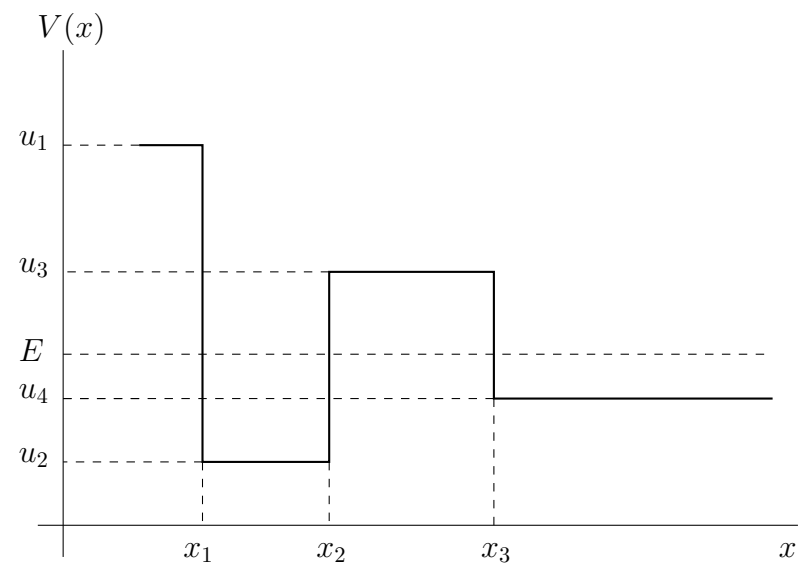

Figure 5: A rectangular potential able to support quasi-bound states as discussed in the text.

\subsection{Calculation of the bound states}

The poles are all contained in $\sin [k L]$, and eigenenergies of the bound states are thus obtained from $\sin \left[k_{n} L\right]=0$, i.e.,

$$
k_{n}=\frac{n \pi}{L}, \quad n=1,2,3, \ldots
$$

From the residues of the Green's function, the correctly normalized eigenfunctions corresponding for the bound states are thus given by

$$
\psi_{n}^{(2)}(x)=\sqrt{\frac{2}{L}} \sin \left(\frac{n \pi x}{L}\right) .
$$

Indeed, these are the exact results for the bound states for the infinite well potential.

\section{Application on quasi-bound states}

In this section, we apply the Green's function approach of previous sections to extract information of systems with quasibound states. A quasi-bound state occurs when a particle move inside a system for a considerable period of time, leaving it when a fairly long time interval $\tau$ has elapsed [29], where $\tau$ is called lifetime of the quasi-bound state. The concept of quasibound states is a fundamental one, and has been applied in all areas of physics. They have been used to calculate tunneling ionization rates [31], to understand the phenomenon of diffraction in time [32], to describe the decay of cold atoms in quasione-dimensional traps [33], and are directly relevant to recent condensed-matter experiments [34].

Let us consider the potential depicted in Fig. 5. Suppose the potentials $u_{1}$ and $u_{3}$ were infinitely high. It would then be possible for particles to be trapped inside the region for $x_{1}<$ $x<x_{2}$, i.e., the system would have genuine bound states, with well defined energy $E>0$. They are genuine bound states in the sense that they are eigenstates of the Hamiltonian with an infinite lifetime. From the Heisenberg uncertainty principle, $\Delta E \Delta t \approx \hbar$, if the energy has null uncertainty its state's lifetime is infinite.

In the situation of a finite barrier as in Fig. 5 (this is rough rectangular approximation for the effective potential in a central

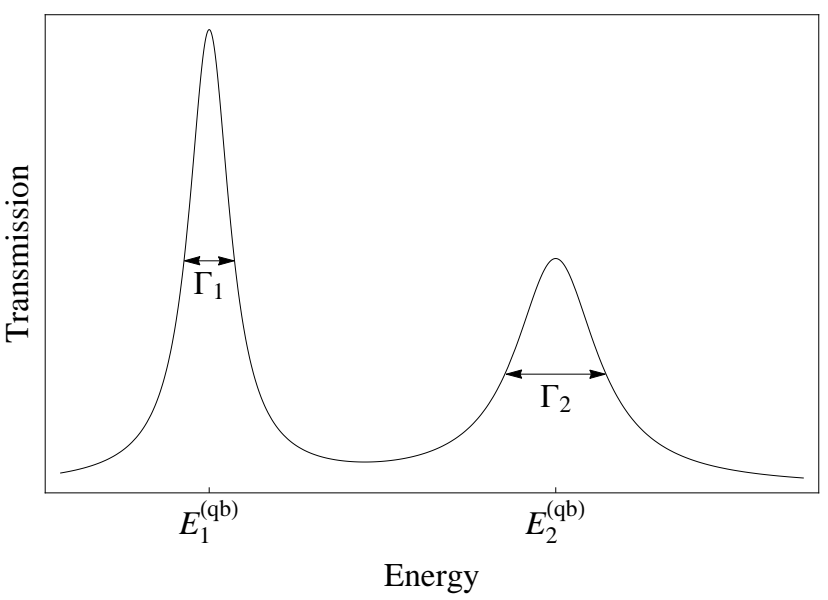

Figure 6: Typical behavior for the transmission coefficient for a potential which has two quasi-bound states with energies $E_{1}^{(\mathrm{qb})}$ and $E_{2}^{(\mathrm{qb})}$ and widths $\Gamma_{1}$ and $\Gamma_{2}$, respectively.

force problem $V(r)$ plus the centrifugal barrier $\left(\hbar^{2} / 2 m\right)[l(l+$ 1) $/ r^{2}$ ] [30]), the particle can be trapped, but it cannot be trapped forever, even if $E<u_{3}$, as a consequence of the tunnel effect. The energy spectrum of these particle will be quasi-discrete, and it consists of a series of broadened levels, whose width in represented by $\Gamma=\hbar / \tau$ [14], and the energy values are called quasi-energies. In the scattering of particles by such potential, the situation becomes very interesting when the incident energy is close to the quasi-energy

$$
E^{(\mathrm{inc})} \approx E^{(\mathrm{qb})} .
$$

In this energy interval, the module square of the transmission amplitude exhibits pronounced peaks, and this is called resonant scattering [30]. In Fig. 6 it is depicted a typical transmission probability as a function of incident energy for a scattering of a potential which supports quasi-bound states.

Now, let us consider the case of a finite square barrier at $x_{2}$ with an infinite barrier at $x_{1}$. In this situation, the system can also has quasi-bound states, due the tunneling through the right square barrier. The scattering eigenfunction for a particle incident from the right is given by

$$
\psi(x) \approx \frac{1}{\sqrt{2 \pi}}\left(e^{-i k_{4} x}+R_{\mathrm{pot}}^{(-)} e^{i k_{4} x}\right),
$$

where $R_{\text {pot }}^{(-)}$is the reflection amplitude of whole potential. By analogy with the previous case, we would try to extract the information from quasi-bound states from the reflection coefficient $R_{\text {pot }}^{(-)}$. Unfortunately, due to the potential to be infinite at left, the reflection coefficient has the value $\left|R_{\text {pot }}^{(-)}\right|^{2}=1$ for all range of energies. Thus, we cannot extract information of quasi-bound states for this kind of potential by the above method So, we propose a Green's function approach to extract information of quasi-bound states for such kind of potential, as we explain below. Following the same steps described in the Section 4 , the Green's function for $x_{i}>x_{3}$ and $x_{1}<x_{f}<x_{2}$ is readily 


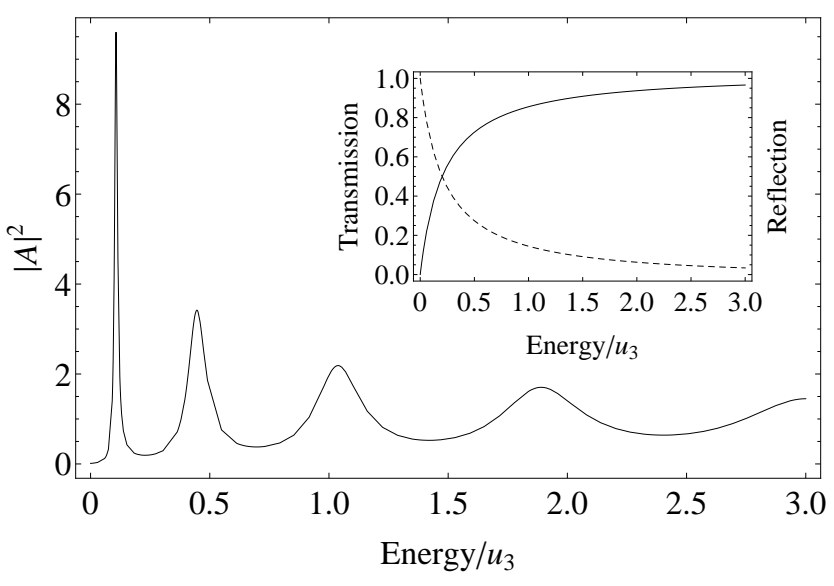

Figure 7: Behavior of $|A|^{2}=\left|T_{b}^{(-)} / \bar{f}_{q b}\right|^{2}$ as function of the energy displaying the presence of quasi-bound states. The parameters are $u_{1}=\infty, u_{2}=u_{4}=0, u_{3}=$ $0.23 \mathrm{eV}, \ell_{3}=80 \AA$ and $\ell_{2}=10.05 \ell_{3}$. In the inset is shown the transmission (solid line) and reflection (dashed line) coefficients for the potential barrier.

obtained and is written as

$$
\begin{aligned}
G_{f, i}= & \frac{m}{i \hbar^{2} \sqrt{k_{2} k_{4}}} \frac{T_{b}^{(-)}}{f_{\mathrm{qb}}} e^{i k_{4}\left(x_{i}-x_{3}\right)} \\
& \times\left(e^{i k_{2}\left(x_{2}-x_{f}\right)}+R_{1}^{(-)} e^{i k_{2}\left(x_{f}+x_{2}-2 x_{1}\right)}\right),
\end{aligned}
$$

with $f_{\mathrm{qb}}=1-R_{1}^{(-)} R_{b}^{(+)} e^{i k_{2} \ell_{2}} \cdot R_{b}^{(+)}$and $T_{b}^{(-)}$are the reflection and transmission amplitudes for the potential barrier given, respectively, by

$$
\begin{gathered}
R_{b}^{(+)}=R_{2}^{(+)}+\frac{T_{2}^{(-)} T_{2}^{(+)} R_{3}^{(+)} e^{2 i k_{3} \ell_{3}}}{1-R_{2}^{(-)} R_{3}^{(+)} e^{2 i k_{3} \ell_{3}}}, \\
T_{b}^{(-)}=\frac{T_{2}^{(-)} T_{3}^{(-)} e^{i k_{3} \ell_{3}}}{1-R_{2}^{(-)} R_{3}^{(+)} e^{2 i k_{3} \ell_{3}}} .
\end{gathered}
$$

For an infinite barrier at $x_{1}$, we have $R_{1}^{(-)}=-1$, and the Eq. (37) simplifies to

$$
G_{f, i}=\frac{2 m}{i^{2} \hbar^{2} \sqrt{k_{2} k_{4}}} \frac{T_{b}^{(-)}}{\bar{f}_{\mathrm{qb}}} e^{i k_{4}\left(x_{i}-x_{3}\right)} \sin \left[k_{2} x_{f}\right],
$$

where $\bar{f}_{\mathrm{qb}}=1+R_{b}^{(+)} e^{i k_{2} \ell_{2}}$. From the interpretation of the Green's function as a probability amplitude (cf. Section 2), we can thus interpret the term

$$
A=\frac{T_{b}^{(-)}}{\bar{f}_{\mathrm{qb}}},
$$

in Eq. (40) as such amplitude. If the potential has quasi-bound states, an incident wave with energy close to the quasi-energy, will have a high probability of tunneling, entering in the trapping region. Consequently, a graph of $|A|^{2}$ as a function of the energy, will have peaks at each energy value close to $E^{(q b)}$. So, we can extract information of quasi-energies and its respective widths from $A$. It is worthwhile to observe that the amplitude $A$ is not normalized, but this is not a problem, because we are only interested in the position of quasi-energies and the width of quasi-states.

For a numerical example, in the Fig. 7 is shown a graph of $|A|^{2}$ as function of energy for the potential in Fig. 5. For the parameters we choose typical values for heterostructures in GaAs [35]. The particle's mass is $m=0.07 m_{e}$, where $m_{e}$ is the electron mass, $u_{1}=\infty, u_{2}=u_{4}=0, u_{3}=0.23 \mathrm{eV}, \ell_{3}=80 \AA$ and $\ell_{2}=10.05 \ell_{3}$. As we can see, it is evident in the graph the existence of quasi-bound states.

The approach presented here for rectangular potentials, can be generalized for smooth potentials, but in this case it is necessary the calculation of the classical action for the quantum particle under the action of the potential. Specifically, we write the amplitude as

$$
A=\frac{T_{b}}{f},
$$

where $T_{b}$ is the generalized transmission amplitude for the smooth barrier between $x_{2}$ and $x_{3}$,

$$
f=1-R R_{\infty} \exp \left[\frac{i}{\hbar} S\left(x_{2}, x_{3} ; E\right)\right]
$$

where $S\left(x_{2}, x_{3}, k\right)$ is the classical action, $R$ is the generalized reflection amplitude for the barrier, and $R_{\infty}$ is the generalized reflection amplitude for the infinity barrier at $x_{1}$. Since $\left|R_{\infty}\right|^{2}=$ 1 , we can write $R_{\infty}=\exp [-i \phi(E)]$, in such way that

$$
f=1-R \exp \left[\frac{i}{\hbar} S\left(x_{2}, x_{3} ; E\right)-i \phi(E)\right] .
$$

All those generalized amplitudes above are obtained by the procedure outlined in $[16,17]$.

\section{Conclusion}

In this work, the exact Green's functions for rectangular single wells are obtained in a rather general way and by a simple method. Our results are the exact ones and, although of the simplicity of the systems considered, Green's functions for such system are not so easy to obtain by standard procedures (for example, solving the inhomogeneous differential equation in Eq. (1)). The procedures allows one to discuss complete arbitrary rectangular single wells and barriers, generalizing and resuming results in the literature. For instance, by withdrawing of the potential step at $x_{1}$ by setting $R_{1}^{(-)}=0$, from Eq. (11) the Green's function for square barrier of Ref. [11, 12] is obtained. The method can be applied for general potentials, including those multidimensional with radial symmetry, but in this case is necessary the calculation of the classical action for the particle under the action of the potential $[16,17]$.

From the poles and residues of the Green's function the bound state eigenenergies and eigenfunctions were obtained with the correct normalization constant. The determination of the later often involves a difficult integral in the other methods.

Finally, we also have discussed an application of the Green's function approach to extract information from quasi-bound states in systems which standard analysis of the quantum amplitudes are not possible. The method could be generalized for smooth 
potentials and applied to the well-known alpha decaying and determination of the dwell times and will be subject of a future work.

\section{Acknowledgments}

The author would like to thank E. O. Silva, C. F. Woellner and J. A. O. Freire, for critical reading the manuscript and helpful discussions. This work was partially supported by the Fundação Araucária (Brazil) under grant number No. 205/2013.

\section{Appendix A. The quantum amplitudes for a step potential}

The step potential is used as building block of our construction. So, in this appendix we just outline the derivation of quantum amplitudes for the step potential. The potential function for the potential step is given by

$$
V(x)= \begin{cases}u_{j} & \text { for } x<0 \\ u_{j+1} & \text { for } x>0\end{cases}
$$

The reflection and transmission amplitudes are obtained from solution of Schrödinger equation. The scattering solutions for the step potential with the incident beam coming from $x=-\infty$ are

$$
\psi(x)= \begin{cases}e^{i k_{j} x}+R_{j}^{(+)} e^{-i k_{j} x} & \text { for } x<0, \\ T_{j}^{(+)} e^{-i k_{j+1} x} & \text { for } x>0,\end{cases}
$$

with $k_{j}=\sqrt{2 m\left(E-u_{j}\right)} / \hbar$. From the matching conditions at the origin, i.e., $\psi\left(0^{-}\right)=\psi\left(0^{+}\right)$and $\psi^{\prime}\left(0^{-}\right)=\psi^{\prime}\left(0^{+}\right)$, we find the sought reflection and transmission amplitudes

$$
R_{j}^{(+)}=\frac{k_{j}-k_{j+1}}{k_{j}+k_{j+1}}, \quad T_{j}^{(+)}=\sqrt{\frac{k_{j+1}}{k_{j}}} \frac{2 k_{j}}{k_{j}+k_{j+1}} .
$$

In the same way, for the case with the incident beam coming from $x=\infty$, we have

$$
R_{j}^{(-)}=-R_{j}^{(+)}, \quad T_{j}^{(-)}=\sqrt{\frac{k_{j}}{k_{j+1}}} \frac{2 k_{j+1}}{k_{j}+k_{j+1}} .
$$

For the case $E>u_{j+1}\left(E<u_{j+1}\right), k_{j+1}$ is a real (imaginary) number and $T_{j}^{( \pm)}$represents the transmission (penetration) amplitude.

\section{Appendix B. Green's function for the infinite well potential from spectral expansion}

In this appendix, we will calculate the Green's function for the infinite well potential from the spectral expansion in Eq. (5). The eigenfunctions and energies for a particle in the infinite well potential are given by

$$
\begin{aligned}
\psi_{n} & =\sqrt{\frac{2}{L}} \sin \left(\frac{n \pi x}{L}\right), \quad n=1,2,3, \ldots, \\
E_{n} & =\frac{n^{2} \pi^{2} \hbar^{2}}{2 m L^{2}}
\end{aligned}
$$

Substituting the eigenfunctions in (B.1) into the spectral expansion of the Green's function, Eq. (5), we arrive at

$$
G_{f, i}=-\frac{4 m L}{\hbar^{2} \pi^{2}} \sum_{n=1}^{\infty} \frac{\sin \left(n X_{f}\right) \sin \left(n X_{i}\right)}{n^{2}+\alpha^{2}},
$$

where $X_{j}=\pi x_{j} / L, \alpha^{2}=-L^{2} k^{2} / \pi^{2}$ and where $k=\sqrt{2 m E} / \hbar$. Using a trigonometric identity for the product of sines, we have

$$
\begin{aligned}
G_{f, i}= & -\frac{2 m L}{\hbar^{2} \pi^{2}}\left\{\sum_{n=1}^{\infty} \frac{\cos \left[n\left(X_{f}-X_{i}\right)\right]}{n^{2}+\alpha^{2}}\right. \\
& \left.+\sum_{n=1}^{\infty} \frac{\cos \left[n\left(X_{f}+X_{i}\right)\right]}{n^{2}+\alpha^{2}}\right\} .
\end{aligned}
$$

The infinite sum above can be evaluated by using the identity 1.445-2 of Ref. [36], and after a straightforward algebra we achieve at

$$
G_{f, i}=-\frac{2 m L}{\hbar^{2} \pi \alpha \sinh [\alpha \pi]} \sinh \left[\alpha\left(\pi-X_{f}\right)\right] \sinh \left[\alpha X_{i}\right] .
$$

Now, by substitution of $X_{j}$ and $\alpha$ and using $\sinh [i \theta]=i \sin [\theta]$, we finally have the Green's function for the infinite well potential

$$
G_{f, i}=\frac{2 m}{\hbar^{2} k \sin [k L]} \sin \left[k\left(x_{f}-L\right)\right] \sin \left[k x_{i}\right]
$$

\section{References}

[1] D. S. Saraga, T. S. Monteiro, Phys. Rev. Lett. 81 (1998) 5796. doi:10.1103/PhysRevLett.81.5796.

[2] C. J. Bolton-Heaton, C. J. Lambert, V. I. Fal'ko, V. Prigodin, A. J. Epstein, Phys. Rev. B 60 (1999) 10569. doi:10.1103/PhysRevB.60.10569.

[3] E. H. Hauge, J. A. Støvneng, Rev. Mod. Phys. 61 (1989) 917. doi:10.1103/RevModPhys.61.917.

[4] F. Capasso, C. Sirtori, J. Faist, D. L. Sivco, S.-N. G. Chu, A. Y. Cho, Nature 358 (1992) 565-567. doi:10.1038/358565a0.

[5] S. Flügge, Practical Quantum Mechanics, Springer-Verlag, Berlin, 1999.

[6] F. L. Scarf, Phys. Rev. 112 (1958) 1137-1140. doi:10.1103/PhysRev.112.1137.

[7] C. Weisbuch, B. Vinter, Quantum Semiconductor Structures: Fundamentals and Applications, Elsevier, 1991.

[8] R. Baltin, Z. Naturforsch 40a (1985) 379-382.

[9] P. Moretti, Phys. Rev. A 46 (1992) 1233-. doi:10.1103/PhysRevA.46.1233.

[10] T. O. de Carvalho, Phys. Rev. A 47 (1993) 2562-. doi:10.1103/PhysRevA.47.2562.

[11] M. A. M. de Aguiar, Phys. Rev. A 48 (1993) 2567-. doi:10.1103/PhysRevA.48.2567.

[12] M. A. M. de Aguiar, Phys. Rev. A 51 (1995) 2654-. doi:10.1103/PhysRevA.51.2654.

[13] M. C. Gutzwiller, Chaos in Classical and Quantum Mechanics, SpringerVerlag, New York, 1990.

[14] E. Merzbacher, Quantum Mechanics, third ed., Wiley, New York, 1998.

[15] M. G. E. da Luz, E. J. Heller, B. K. Cheng, J. Phys. A 31 (1998) 2975. doi:10.1088/0305-4470/31/13/007.

[16] M. G. E. da Luz, B. K. Cheng, M. W. Beims, J. Phys. A 34 (2001) 5041. doi:10.1088/0305-4470/34/24/303.

[17] F. M. Andrade, B. K. Cheng, M. W. Beims, M. G. E. da Luz, J. Phys. A 36 (2003) 227. doi:10.1088/0305-4470/36/1/315.

[18] B. R. Holstein, Am. J. Phys. 51 (1983) 897. doi:10.1119/1.13374.

[19] M. G. Rozman, P. Reineker, R. Tehver, Phys. Rev. A 49 (1994) 33103321. doi:10.1103/PhysRevA.49.3310. 
[20] A. G. M. Schmidt, B. K. Cheng, M. G. E. da Luz, J. Phys. A 36 (2003) L545. doi:10.1088/0305-4470/36/42/L01.

[21] A. G. M. Schmidt, B. K. Cheng, M. G. E. da Luz, Phys. Rev. A 66 (2002) 062712. doi:10.1103/PhysRevA.66.062712.

[22] F. M. Andrade, M. G. E. da Luz, Phys. Rev. A 84 (2011) 042343-. doi:10.1103/PhysRevA. 84.042343.

[23] F. M. Andrade, M. G. E. da Luz, Phys. Rev. A 86 (2012) 042309doi:10.1103/PhysRevA.86.042309.

[24] E. N. Economou, Green's Functions In Quantum Physics, third ed., Springer-Verlag, Germany, 2006.

[25] L. S. Schulman, Techniques and Applications of Path Integrals, Dover Publications, Mineola, New York, 2005.

[26] H. Kleinert, Path Integrals in Quantum Mechanics, Statistics, Polymer Physics, and Financial Markets, fourth ed., World Scientific Publishing Company, Singapore, 2006.

[27] H. Kleinert, I. Mustapic, J. Math. Phys. 33 (1992) 643-. doi:10.1063/1.529800.

[28] A. R. H. Richard P. Feynman, Quantum Mechanics and Path Integrals: Emended Edition, Dover Publications, 2010.

[29] L. D. Landau, E. M. Lifschitz, Quantum Mechanics, Pergamon, Oxford, 1981.

[30] J. J. Sakurai, J. Napolitano, Modern Quantum Mechanics, 2nd ed. ed., Addison-Wesley, 2011.

[31] G. N. Gibson, G. Dunne, K. J. Bergquist, Phys. Rev. Lett. 81 (1998) 2663 doi:10.1103/PhysRevLett.81.2663.

[32] E. Torrontegui, J. Muñoz, Y. Ban, J. G. Muga, Phys. Rev. A 83 (2011) 043608. doi:10.1103/PhysRevA. 83.043608.

[33] A. del Campo, J. G. Muga, Europhys. Lett. 74 (2006) 965. doi:10.1209/epl/i2006-10061-5.

[34] M. Sato, H. Aikawa, K. Kobayashi, S. Katsumoto, Y. Iye, Phys. Rev. Lett 95 (2005) 066801. doi:10.1103/PhysRevLett.95.066801.

[35] A. P. Jauho, M. Jonson, J. Phys.: Condens. Matter 1 (1989) 9027. doi:10.1088/0953-8984/1/45/025

[36] I. S. Gradshteyn, I. M. Ryzhik, Table of Integrals, Series, and Products, seventh edition ed., Academic Press, 2007. 